\title{
Índice de diversidad biológica urbana de la ciudad de La Antigua Guatemala
}

\author{
Jorge García-Polo*, Fernando Castillo-Cabrera, José J. Vega \\ Centro de Estudios Conservacionistas, Facultad de Ciencias Químicas y Farmacia, \\ Universidad de San Carlos de Guatemala, Guatemala.
}

*Autor al que se dirige la correspondencia: jjgp78@yahoo.com

Recibido: 27 de agosto 2015 / Revisión: 04 de noviembre 2015 / Aceptado: 15 de febrero 2016 / Disponible en línea: 01 de agosto 2016

\section{Resumen}

T a urbanización es un fenómeno global cuyo pronóstico prevé que para el 2050 hasta el $70 \%$ de la población Lmundial vivirá en ciudades. Esto tendrá un impacto sobre la diversidad biológica, lo que podría generar pérdida de especies y de los servicios ecosistémicos relacionados. Las administraciones municipales deben contemplar este componente para la planificación y desempeño ambiental de sus ciudades y áreas urbanas. El Convenio sobre la Diversidad Biológica (CDB) ha planteado una nueva herramienta para estimar la diversidad biológica en ciudades: el índice de diversidad biológica urbana. En este proyecto se evaluó este índice para la ciudad de La Antigua Guatemala. De un máximo de 72 puntos, el resultado para la ciudad de La Antigua Guatemala fue de 33 puntos. Se midieron 18 indicadores de tres componentes: (1) biodiversidad urbana con un desempeño alto, (2) servicios ecosistémicos con un desempeño bajo y (3) gobernanza y manejo de la diversidad biológica con un desempeño medio. Los registros para la línea base de la biodiversidad son: 99 especies de aves, 148 especies de plantas vasculares, 46 especies de mariposas y 11 especies de murciélagos. Se recomienda a las autoridades de la ciudad de La Antigua Guatemala y actores involucrados, elaborar de urgencia la estrategia local de diversidad biológica y su plan de acción.

Palabras claves: Ecología urbana, infraestructura verde, gobernanza ambiental, servicios ecosistémicos urbanos, Convenio sobre la Diversidad Biológica

\begin{abstract}
$\mathrm{T}$ The global process of urbanization predicts a $70 \%$ of all world citizens living in cities by the year 2050 . The result will be a high impact on biological diversity like species loss and degradation of ecosystem services. Local governments have to introduce this component in the environmental planning and performance of their cities. The UN Convention on Biological Diversity (CBD) has proposed a new tool to evaluate the biological diversity in cities: The City Biodiversity Index (CBI). In this project the CBI for the city of La Antigua Guatemala was assessed. The city scores 33 points out of 72 . The indicators measured were related to three main components: (1) biological diversity with high value, (2) ecosystem services with low value and (3) governance and management of biological diversity with medium value. Biodiversity base line records were: 99 bird species, 148 plant species, 46 butterfly species and 11 bat species. The project recommends the urgent elaboration of Antigua Guatemala's City Local Biodiversity Strategy and Action Plan (LBSAP).
\end{abstract}

Keywords: Urban ecology, green infrastructure, environmental governance, urban ecosystem services, Convention on Biological Diversity 


\section{Introducción}

Los datos de la Organización de las Naciones Unidas (ONU), indican que la población urbana del mundo para el año 2050 corresponderá al $67.1 \%$ de la población mundial total. Para Guatemala, el $68.5 \%$ de la población se estima que habitará en zonas urbanas (United Nations, 2012). Esto significa que siguiendo el mismo patrón de desarrollo urbano del país, habrá impactos en el ambiente y en la diversidad biológica. Esta tendencia le impone retos a la administración pública y a todos los sectores de la población para la búsqueda de modelos que permitan la coexistencia de ciudades y centros urbanos con la naturaleza.

El Consejo Nacional de Áreas Protegidas (Conap) define la diversidad biológica como: "la variación de las formas de vida del planeta; al referirnos a sus componentes, hacemos alusión a tres diferentes escalas con atributos propios, siendo estos: diversidad genética, diversidad de especies y diversidad de ecosistemas" (2013). La información sobre el desempeño ambiental de las ciudades y de la diversidad biológica urbana en el país es escasa, los servicios ecosistémicos que proveen, y la falta de reconocimiento de éstos en la planificación ambiental urbana, puede observarse en las prácticas de gobernanza y ordenamiento de las áreas urbanas. Según el Convenio para la Diversidad Biológica (CDB), estos elementos son clave en el desempeño ambiental de las ciudades y por lo mismo fueron incluidos en el cálculo de un índice que valora el papel de la diversidad biológica en las ciudades (Chan et al., 2014). Este índice fue propuesto en la Reunión mundial de ciudades en Singapur en 2008, por el entonces alcalde de la ciudad, dada la importancia que tienen las ciudades para alcanzar los objetivos del CDB. El desarrollo de este índice para la ciudad de La Antigua Guatemala, será una herramienta crítica de evaluación, para que la planificación ambiental mejore las estrategias de sustentabilidad y para mantener la calidad de vida en la ciudad.

\section{Materiales y métodos}

Se aplicó el índice de diversidad biológica urbana para la ciudad de La Antigua Guatemala. El índice consiste en 23 indicadores según esquema de la Figura 1 y está constituido de 3 componentes:

- Componente I: Diversidad biológica en la ciudad, con 10 indicadores;

- Componente II: Servicios ecosistémicos que provee la diversidad biológica en la ciudad, con 4 indicadores; $y$

- Componente III: Gobernanza y manejo de la diversidad biológica en la ciudad, con 9 indicadores.

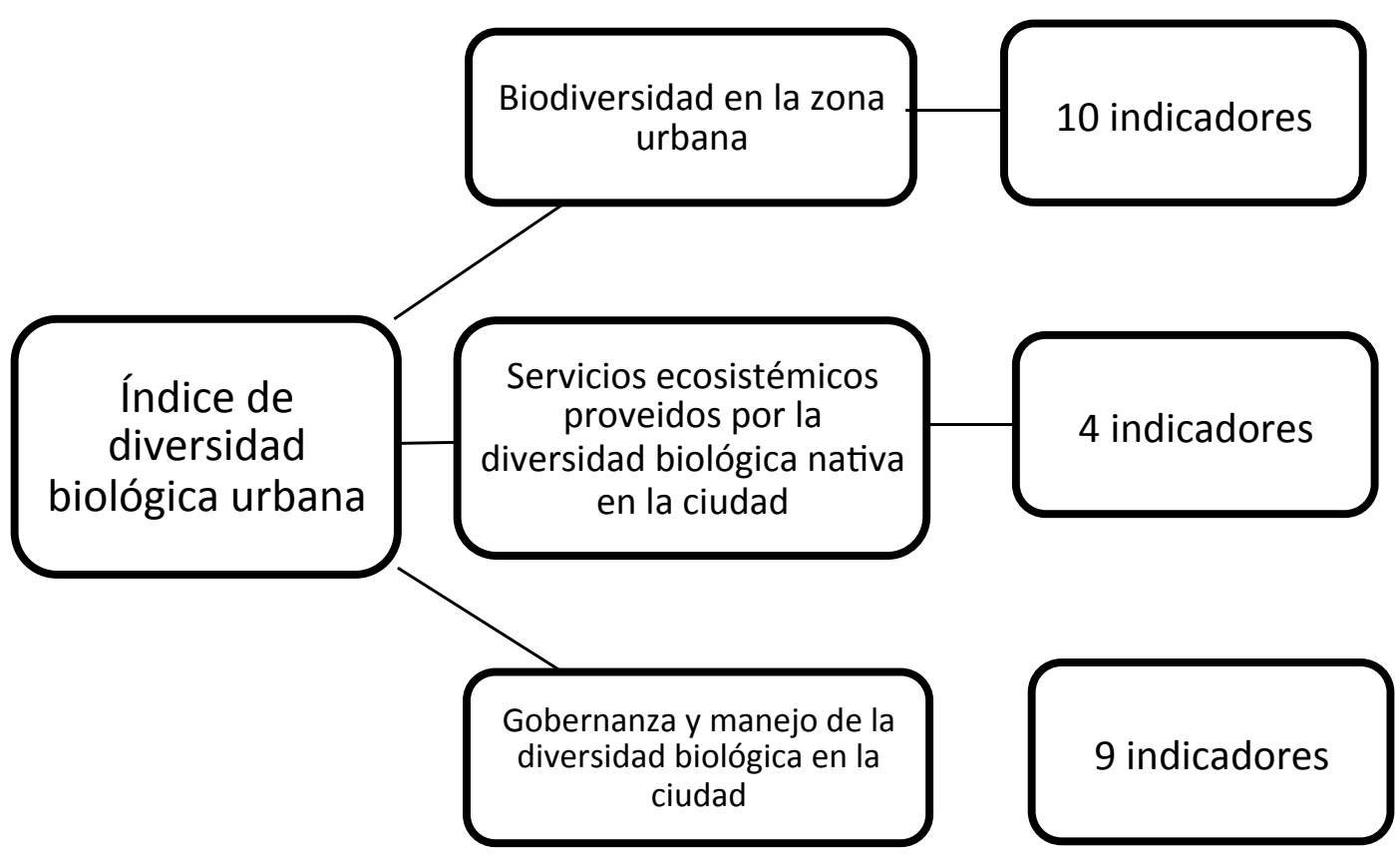

Figura 1. Componentes del índice de diversidad biológica urbana. 
Los criterios para evaluar cada indicador se basaron en el Manual de usuarios del índice de diversidad biológica urbana (versión del 25 de enero de 2013), publicado en el año 2014 (Chan et al., 2014). Por ser la primera vez que se aplica el índice, se consideró para la Ciudad de La Antigua Guatemala realizar la línea base, calificándose 18 indicadores. Cada uno de éstos se estimó con un valor máximo de 4 puntos, para obtener una sumatoria de 72 puntos total.

Los indicadores 4-8 fueron registrados y serán utilizados para calcular el cambio en el número de especies (se recomienda aplicar el índice cada tres años para calcular estos indicadores). En el índice se propone el estudio de las especies de: aves, plantas vasculares, mariposas diurnas y otros grupos taxonómicos. En el presente estudio se estableció la línea base de los grupos propuestos y además se estudió como grupo adicional la diversidad de murciélagos. El sitio de estudio incluyó los siguientes hábitats urbanos y periurbanos: parques, monumentos, cafetales y bosques. Se estudiaron las especies en cuatro localidades de cada hábitat. El sitio de estudio se muestra en la Figura 2, debido a la falta de límites oficiales de la ciudad, se utilizó en un área adicional de $3 \mathrm{~km}$ de la zona núcleo.

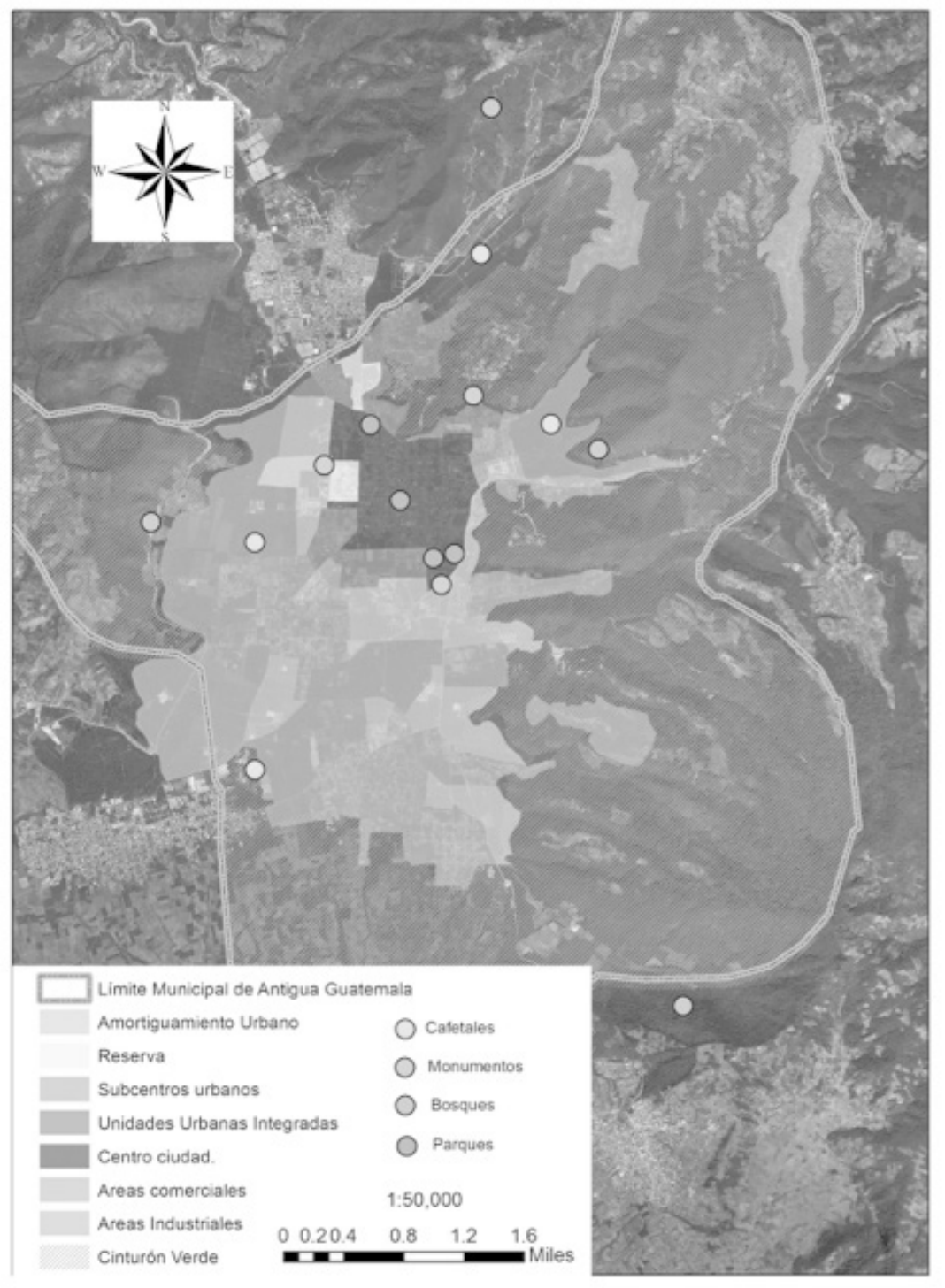

Figura 2. Mapa ubicación de los sitios de estudio. 
Se realizaron inventarios de las especies de plantas vasculares en cada hábitat. Las especies de aves se registraron en cada hábitat por medio de puntos de conteo, a través de observación e identificación de cantos. Las mariposas fueron colectadas con redes entomológicas (horas / red) en cada uno de los hábitat. Para la captura de murciélagos se utilizaron redes de niebla. Posterior a su registro todos los murciélagos capturados fueron liberados. Las muestras de plantas fueron depositadas en el Herbario de la Universidad de San Carlos de Guatemala (Herbario USCG) del Centro de Estudios Conservacionistas (número de ingreso 40260 - 40425) y las mariposas colectadas fueron depositadas en la Colección del Museo de Historia Natural, Universidad de San Carlos de Guatemala (Usac).

Los cálculos para generar cada indicador y los criterios de calificación se muestran en el Anexo 1. Posteriormente, se realizó la sumatoria de la calificación de cada indicador para obtener el índice para La Antigua Guatemala. Además, el resultado del índice para la ciudad de estudio se comparó con los índices de otras ciudades del mundo. Los datos fueron proporcionados por el equipo de Singapur. Se calificó cada indicador con los mismos criterios y se utilizaron los porcentajes del total para fines comparativos.

\section{Resultados}

El CBI urbano fue calculado por medio de la medición de los indicadores de tres componentes, obteniéndose un total de 33 puntos de un máximo de 72 puntos. La Tabla 1 muestra los valores obtenidos para cada componente y sus indicadores. El resultado consiste en la suma de las calificaciones de 18 indicadores, la calificación de cada indicador se presentan en la Figura 3. El valor del índice está por debajo de lo previsto al inicio del estudio.

La evaluación de los indicadores ha tenido obstáculos en otros estudios de ciudades, por ejemplo: la falta de claridad en los límites de la ciudad (Kohsaka et al., 2013). En el tema de gobernanza, la ciudad muestra un desempeño medio. Algunos indicadores tienen la calificación más alta y otros con una calificación de cero. Es importante vincular más instituciones, tener más actividades y especificar montos para actividades directamente relacionada con diversidad biológica.

En general la ciudad debe de generar su estrategia y plan de acción de local de diversidad biológica y con esto tener un mejor desempeño en el índice CBI. Para la línea base de diversidad biológica de la ciudad de La Antigua Guatemala se registraron en el sitio de estudio: 99 especies de aves, 148 de plantas vasculares, 46 de mariposas diurnas y 11 de murciélagos.

Según se propone en el Manual del CBI, los 23 indicadores antes mencionados deben ser evaluados tres años después de esta línea base. Con eso será posible calcular y calificar los indicadores del 4 al 8 del componente I. Además, los resultados del índice para La Antigua Guatemala fueron comparados con otras ciudades del mundo.

La Figura 4 muestra el valor del índice en porcentajes del total (debido a que el número de indicadores medidos en las ciudades es variable). Para ninguna ciudad se consideraron los indicadores 4 al 8 del componente I, debido a que ninguna ciudad presentaba el resultado del cambio en número de especies, sino únicamente el número de especies de la línea base.

Tabla 1

Resultados del índice por sus tres componentes e indicadores, La Antigua Guatemala 2013

Indicadores

Calificación

Subtotal Componente I

1) Proporción de áreas naturales en la ciudad de La Antigua Guatemala. Total del área terrestre $=253.4$ Ha Total del área verde (permeable) $=84.3$ ha (el verde urbano) Proporción: 33.3\%

2) Conectividad para detener fragmentación. EMS* calculado de 614.12 Ha, para obtener este índice se utilizó un área adicional de $3 \mathrm{~km}$ de la zona núcleo de la ciudad (sitio de estudio).

3) Biodiversidad nativa en áreas construidas (aves): 46 especies nativas de aves dentro de la zona núcleo (áreas construidas) de la cuidad de La Antigua Guatemala.

4) Cambio en el número de especies nativas en la ciudad: Aves.

Se registraron 99 especies de aves en el sitio de estudio (línea base). 
Tabla 1, continuación

Indicadores

Calificación

5) Cambio en el número de especies nativas en la ciudad: Plantas.

Se registraron 148 especies de plantas en el sitio de estudio (línea base).

$\mathrm{NSC}^{* *}$

6) Cambio en el número de especies nativas en la ciudad: Mariposas.

Se registraron 46 especies de Mariposas en el sitio de estudio (línea base).

NSC**

7) Cambio en el número de especies nativas en la ciudad: Murciélagos.

Se registraron 11 especies de murciélagos en el sitio de estudio (línea base).

$\mathrm{NSC} * *$

8) Cambio en el número de especies nativas en la ciudad: Insectos y otros (pendiente).

$\mathrm{NSC} * *$

9) Proporción de áreas protegidas: No hay áreas protegidas. Dentro del área de estudio.

10) Proporción de especies invasoras.

3

Subtotal Componente II

11) Regulación del agua. Resultado: 33\%

3

12) Regulación climática y secuestro de carbono.

2

13) Servicios de recreación natural y educación ambiental (área/1000 personas): Existen 2.37 has ó $23,718.065 \mathrm{~m}^{2}$ de parques naturales (pero sin área natural sino con vegetación manejada) 52,700 habitantes. 0.045 ha por cada mil habitantes.

14) Servicios de recreación natural y educación ambiental (visita educacional): No se realizan visitas a áreas protegidas en el año.

Subtotal Componente III

15) Presupuesto destinado a diversidad biológica.

16) Número de proyectos sobre biodiversidad x autoridades: Menos de12 por año.

17) Políticas, reglas y regulaciones-estrategias y planes de acción locales sobre biodiversidad: No se ha implementado ninguna.

18) Capacidad institucional. Número de funciones relacionadas a biodiversidad: Vivero municipal.

19) Capacidad institucional. Número de agencias de la ciudad o del gobierno local que participan en la cooperación entre organismos relacionados con la materia de biodiversidad: Más de 5.

20) Participación y asociación. Existencia y estado de proceso de consulta pública formal o informal referente a los asuntos relacionados con la biodiversidad. Existe un proceso formal.

21) Participación y asociación. Número de entidades/empresas privadas/ONG/instituciones académicas/organizaciones internacionales con las que la ciudad se está asociando en las actividades de biodiversidad, proyectos y programas. Entre 7 a 12 instituciones.

22) Educación y sensibilización (currículo escolar). Se ha incluido la educación ambiental dentro de currículo escolar oficial. Se ha intentado incluir temas de diversidad biológica.

23) Educación y sensibilización (eventos). De 60 -149 eventos realizados por /1,000 personas/año.

Total

Nota. *EMS: Métrica del Paisaje (Efective Mesh Size). **NSC: No se calculó (Los datos obtenidos conforman la línea base, el cálculo del cambio de especies se recomienda realizarlo tres años después de la línea base). 


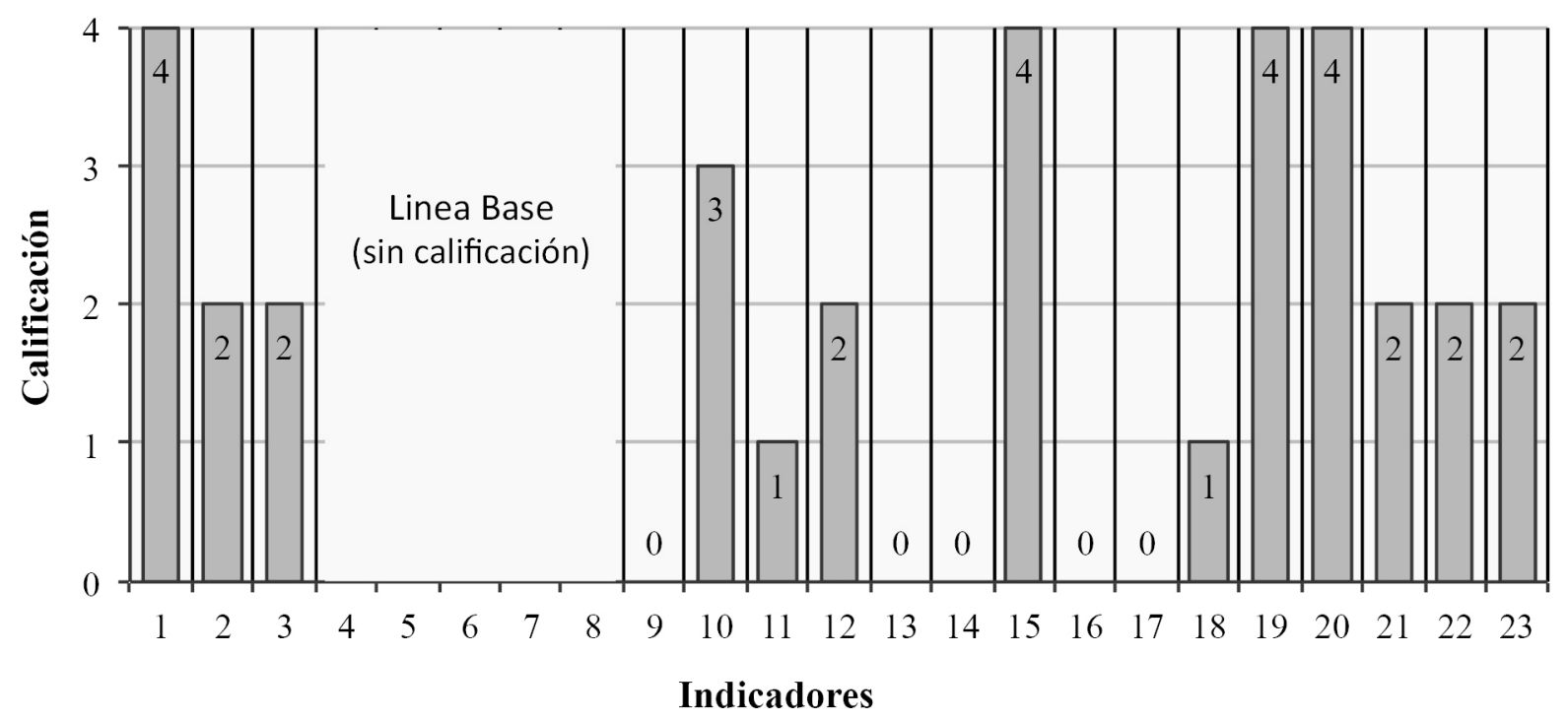

Figura 3. Calificación por indicador del índice de diversidad biológica urbana, de la ciudad de La Antigua Guatemala 2013.

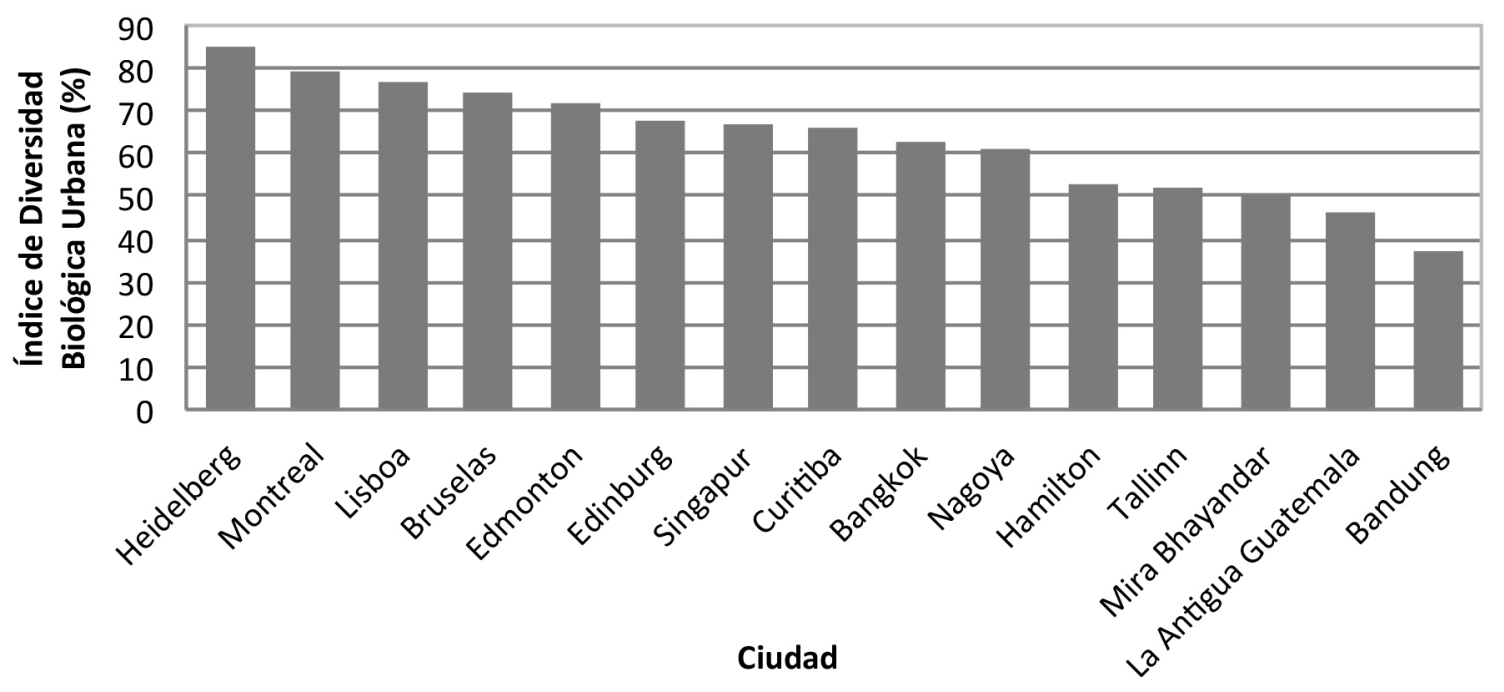

Figura 4. Comparación del índice de La Antigua Guatemala con otras ciudades del mundo. 


\section{Discusión}

\section{La diversidad biológica nativa en la ciudad de Antigua Guatemala}

El componente I para la ciudad de La Antigua Guatemala presentó una calificación alta. Se observó que el límite de la ciudad no está oficialmente definido, lo cual es un obstáculo para la medición de los indicadores. Además, Chan, L. (comunicación personal, 16 de octubre, 2013) autora principal del manual de usuarios, recomendó que por su naturaleza, los indicadores del 4 al 8 se midieran pero que no recibieran calificación, ya que conformarán la línea base del índice. Estos indicadores se calcularán cuando se realice nuevamente la evaluación del índice, 3 años después de ésta evaluación, y tener un punteo que se contabilizará para el total. El indicador 1 (proporción de áreas verdes) fue calculado para la zona núcleo de la ciudad, basado en los resultados de Castillo, García, López y Celada (2013). Sin embargo, al no existir un límite establecido de la ciudad, este valor es sensible a la escala. Para mejorar el desempeño en este indicador, es necesario incrementar la proporción de áreas naturales públicas. $\mathrm{Al}$ incrementar las áreas naturales, es importante mejorar la conectividad entre las áreas verdes en la ciudad, lo cual es necesario según lo documentado con el indicador 2. Para esto se requiere evaluar la conectividad de forma funcional, es decir, el papel de las áreas verdes en la movilidad de diferentes grupos taxonómicos.

En el caso de especies nativas en áreas construidas o zona núcleo de la ciudad (indicador 3), se tomaron en cuenta las especies de aves presentes en monumentos y parques de la ciudad, registrando un número de 46 especies de aves. Este número se considera aceptable, pero se recomienda que se haga un esfuerzo mayor, debido a que en el estudio solamente se logró trabajar en época lluviosa. Es probable que realizando observaciones durante la época seca, puedan surgir otras especies, incluyendo algunas de carácter migratorio. Como se indicó anteriormente, el indicador 4 a 8 sobre el cambio en número de especies, en esta ocasión se estableció la línea base, por lo que en tres años podrá ser estimado. Para la línea base se registraron en todo el sitio de estudio: 99 especies de aves, 148 especies de plantas, 46 especies de mariposas diurnas y 11 de murciélagos. Es importante mencionar que en un estudio anterior (Kraker-Castañeda \& Pérez-Consuegra, 2011) se reportaron 12 especies de murciélagos, lo que podría indicar que la variación del entorno ecológico se mantiene estable.

El indicador 9 sobre áreas protegidas dentro de la ciudad, obtuvo un punteo de 0 puntos, debido a la falta de áreas protegidas dentro de La Antigua Guatemala. En Guatemala, el Conap, ha exhortado la creación de áreas protegidas dentro de cascos urbanos y municipales como una forma de beneficiar a los vecinos con espacios para la recreación. Además, el tema ha sido incentivado internacionalmente. La Unión Internacional para la Conservación de la Naturaleza (UICN), ha formado un grupo de especialistas enfocados en áreas protegidas urbanas. Las áreas protegidas urbanas son importantes porque promueven la calidad de vida y la salud humana, conectan a las personas con la naturaleza y le dan un sentido de pertenencia, son un espacio para aprender de la naturaleza y la biodiversidad, proveen de servicios ecosistémicos, contribuyen a la infraestructura verde en la ciudad, participan en la mitigación al cambio climático, refuerzan la resiliencia frente al cambio climático, protegen especies que no se protegen en otros lados y apoyan a la economía local (Trzyna, 2014).

Sobre indicador 10, las especies invasoras encontradas fueron 3; una especie de ave y dos de plantas. En el caso del ave invasora, la especie es Passer domesticus (gorrión europeo). Sin embargo, aún y cuando es catalogada como especie invasora en México (Comité Asesor Nacional sobre Especies Invasoras, 2010), en Guatemala se encuentra como especie de la Lista gris de especies exóticas (Conap, 2011). En el caso de las plantas, las especies invasoras son Taraxacum officinale L.) Weber ex F.H.Wigg y Ricinus communis L. conocidas como diente de león y ricino, respectivamente. A nivel nacional la primera se encuentra en la Lista gris (Conap, 2011) y la segunda no se reporta. Otros países si las reportan como especies invasoras, tal es el caso del ricino que es catalogada como altamente invasora, probablemente por su alta plasticidad (Martins, Haddad, \& Semir, 2011). Para el caso del diente de león, se reporta como invasora desde EEUU hasta Chile, lo que podría significar que en Guatemala también sería invasora. Ésta planta compite por los polinizadores y además es resistente a herbicidas (Quiroz, Pauchard, Marticorena, \& Cavieres, 2009).

Esta falta de información sobre especies invasoras abre una importante ventana a la investigación en Guatemala, porque representan un peligro para las especies nativas y deben tener un control para evitar cambios en las interacciones ecológicas. 
Los servicios ecosistémicos que provee la diversidad biológica nativa en la ciudad

Como se observa en los resultados obtenidos en esta investigación, la ciudad de La Antigua Guatemala tiene un desempeño bajo en estos indicadores. Además estos son reconocidos por la ciudadanía como importantes, ya que sus beneficios son identificados (Castillo et al., 2013). Esto puede interpretarse como un aspecto negativo de la ciudad y de sus habitantes, debido que el valor sugiere que la provisión de estos servicios, de acuerdo al indicador, no está en un nivel básico (beneficios en materia de regulación del agua, del clima y de la captura de carbono, además de los servicios relacionados a educación y recreación).

En general se evidencia que existe insuficiente espacio de áreas verdes (naturales o manejadas) que permitan mejorar estos indicadores. La ciudad no posee más espacios en propiedad y los que quedan están en diferentes regímenes de propiedad como el Consejo para la Protección de Antigua Guatemala, empresas privadas y personas individuales. Por lo tanto, es necesario definir estrategias para buscar soluciones basadas en la naturaleza (Eggermont et al., 2015) lo que supone reconocer la ciudad como un sistema socio-ecológico que debe resolver los retos de la sociedad mediante el uso sostenible de la naturaleza maximizando el suministro de servicios ecosistémicos.

Hay que reconocer que también la falta de límites oficiales sobre el área que debería ocupar la ciudad, puede modificar el dato obtenido. Al aumentar los límites de la ciudad, es probable que los indicadores 11 y 12 tengan mayor punteo. La falta de claridad en los límites también es un problema a nivel mundial encontrado en otras ciudades que han llevado a cabo el análisis del índice (Kohsaka et al., 2013) por lo que, tal y como se ha observado en otros estudios, la municipalidad de La Antigua Guatemala debería realizar consultas para demarcar los límites de la ciudad, o bien, realizar cambios en el cálculo del indicador. Esta sugerencia ha sido planteada por otros estudios que han encontrado que servicios ecosistémicos (como los indicadores 11 y 12) provienen de áreas alejadas de los límites de la ciudad. Puesto que las ciudades al ser grandes centros de consumo, afectan regiones más allá de su espacio (tanto regional como globalmente) (Kohsaka et al., 2013).

Para el caso de los indicadores 13 y 14 relacionados al tema de recreación y educación, se puede interpretar esto en dos direcciones: la primera, relacionada siempre al tema de la falta de espacios naturales o áreas verdes públicas para que sean visitadas por las personas. Es decir, existe insuficiente espacio para que las personas hagan actividades de recreación. De igual manera que los indicadores anteriores, es lo que puede hacer la administración municipal, pero también está el efecto de los límites y de la escala en el cálculo de los indicadores. Por ejemplo, la Finca Florencia, (que está en otro municipio cercano pero es administrada por la municipalidad de La Antigua Guatemala) es visitada por numerosos vecinos, no sólo de la ciudad estudiada, sino también de municipios cercanos, además de ser frecuentemente visitada por habitantes de la ciudad capital. Esto es un problema que debería ser comunicado a los encargados de ir buscando mejoras en el cálculo del índice. Como ejemplo, Kohsaka y colaboradores (2013) encontraron en general para varias ciudades evaluadas, Edmonton, Mira Bhainder, Lisboa, dos ciudades de Japón, existe un problema en cómo definir las áreas naturales que mejor sería realizar un análisis de gradiente lo que significa, expandir la escala.

La segunda dirección está relacionada más con el quehacer de la municipalidad y otras instituciones para facilitar y promover las visitas de estudiantes de todos los niveles a diferentes áreas verdes tanto naturales como manejadas. De manera general se consultó a la delegación departamental de Educación de Sacatepéquez y ellos no autorizan viajes o salidas de escolares fuera de cierto rango. Con ello los escolares pierden la oportunidad de tener contacto con la diversidad biológica del país. Una coordinación más efectiva entre los diversos grupos de la sociedad civil y las instituciones de gobierno junto con la municipalidad, podrían revertir este patrón encontrado.

Sin embargo, ambas conllevan la responsabilidad de claramente realizar una delimitación de los límites de lo que se podría considerar la ciudad de La Antigua Guatemala, o al menos, utilizar límites relacionados a los servicios ecosistémicos que, ya como se mencionó anteriormente, tienen una dimensión espacial regional y hasta global. Asimismo, la decisión de tener más espacio público y privado verde, y hasta con carácter protegido, podría significar un aumento de los beneficios a los habitantes de la ciudad y como consecuencia, mejorar el desempeño ambiental en este componente del índice. De acuerdo a la revisión del índice para varias ciudades y estudios anteriores (Haase, Schwarz, Strohbach, Kroll, \& Seppet, 2012; Kohsaka et al., 2013; Martín-López et al., 2012; Raudsepp-Hearne, Peterson, \& Bennett, 2010) los servicios ecosistémicos aparecen en grupos y tienen interacciones entre ellos (sinergias, 
compensaciones, pérdidas) por lo que se recomienda que en este índice se evalúen también estas características, a mediano y largo plazo y en una escala espacial regional. Especialmente evaluar los efectos de interacción en los cafetales cercanos a la ciudad, pues en estos tipos de agro-ecosistemas se han encontrado efectos de interacción relacionados a las prácticas de manejo (Boreaux, Kushalappa, Vaas, \& Ghazoul, 2013) y su impacto sobre la ciudad no se ha estimado.

Además, en nuestro estudio se identificó que los cafetales, como un tipo de área verde, son ambientes relevantes para proveer servicios ecosistémicos a la ciudad. Para mejorar el desempeño de la ciudad en tema de servicios ecosistémicos, será necesaria la vinculación de sociedad civil, fincas de café y gobierno local, con el objetivo de evaluar cuantitativamente (oferta y demanda) los distintos servicios que se obtienen de estas áreas así como su valoración económica, tal y como se ha realizado en otros países con cultivos de café (Taugordeau et al., 2014; Berghöfer, 2011).

\section{Gobernanza y manejo de la diversidad bioló- gica en la ciudad}

El componente III de la ciudad tiene indicadores relacionados a la administración, gestión, planificación y diseño de las ciudades, es decir, la gobernanza relacionada a la diversidad biológica en la ciudad. En este aspecto la ciudad muestra un desempeño medio. Algunos indicadores tienen el punteo alto y otros con punteo de cero.

En el caso del indicador 15 y 16 (presupuesto y proyectos sobre biodiversidad, respectivamente) los cuales están directamente relacionados, se observa una contradicción muy evidente: se tiene un presupuesto que de acuerdo al CBI es adecuado para temas ambientales en general. Pero al evaluar la información sobre los proyectos específicos al tema de diversidad biológica, estos son muy escasos y la diversidad biológica aparece en un plano secundario.

Este problema, que los presupuestos son generales y no evidencian acciones directas sobre la diversidad biológica, se ha reportado para otras ciudades, en donde, debido a la estructura administrativa, es difícil especificar los montos exactos para tales acciones (Kohsaka et al., 2013). Los proyectos que la municipalidad de la Antigua Guatemala tiene en relación a la diversidad biológica son escasos y una forma de mejorar en este aspecto sería de convocar a todos los sectores involucrados para planificar y diseñar una estrategia local y un plan de acción local de biodiversidad, recomendado por el CDB. En unas ciudades de Japón se implementó la norma ISO 14001 y tuvo un efecto positivo en la administración de la ciudad, haciendo que todos los departamentos (que en general desconocen el tema de diversidad biológica, salvo los relacionados al tema ambiental) tuvieran acceso a la importancia de la gestión y manejo de la diversidad biológica en la ciudad (Kohsaka et al., 2013). Esta misma situación podría recomendarse para la ciudad de la Antigua Guatemala. Esto básicamente, significa que la diversidad biológica constituya un eje transversal en el quehacer de las autoridades a nivel local, y en este caso de las ciudades (Avlonitis et al., 2013)

También con el indicador 17 ocurre un desconocimiento de cierta normativa internacional suscrita por el país en materia de diversidad biológica. Las acciones que recomienda el CBD no han sido implementadas por la ciudad de Antigua Guatemala. Una lectura cuidadosa de estos compromisos de Estado, y que benefician a los habitantes y turistas en la ciudad, (además de mejorar el manejo y gestión de la diversidad biológica), es necesaria para realizar acciones locales sobre este tema. Se deben desarrollar actividades participativas para evaluar la política ambiental de la municipalidad e implementar las acciones recomendadas como la estrategia local y plan de acción sobre diversidad biológica (Avlonitis et al., 2013).

En cuanto a las funciones institucionales relacionadas a diversidad biológica (indicador 18) también la ciudad obtuvo un punteo bajo. Esto se debe a la falta de implementación de acciones recomendadas en el Plan Maestro de la Ciudad (López García \& Martín Hernández, 2010) tales como mariposarios, jardines botánicos y otras que visibilizarían el papel fundamental de la diversidad biológica en la vida de las personas. Estas acciones además cumplirían un papel educador para los diferentes sectores sociales locales y regionales. Otras ciudades (Figura 4) han tenido un punteo alto porque tienen diferentes instituciones realizando funciones relacionadas a la diversidad biológica. Por ejemplo, las ciudades de Bangkok y Nagoya poseen instituciones como museos, jardines botánicos, centros de investigación, centros de educación ambiental y diversidad biológica (Secretariat of the Convention on Biological Diversity, 2012).

En relación al indicador 19, la cooperación de otras agencias o instituciones de gobierno con la municipalidad para realizar acciones concretas en diversidad biológica, obtuvo el punteo máximo de 4 puntos. Sin 
embargo, deben de vincularse más agencias y especificar las acciones. Esto fortalecería a las partes involucradas en uso eficiente de los recursos, transmisión de conocimiento y capacidad técnica.

Los indicadores 20 y 21 sobre participación y asociación, en cuanto a procesos formales de consulta, son implementados como parte del proceso rutinario en cualquier tipo de proyecto que pueda tener un impacto ambiental. Además, son necesarias acciones y procesos locales para evaluar impactos en la diversidad biológica y servicios ecosistémicos por medio de mecanismos participativos. En cuanto, a consorcios formales o informales de la ciudad con otras entidades (agencias nacionales, subnacionales, empresas privadas, sociedad civil), es necesario formalizar las acciones que realiza la municipalidad, por ejemplo como las acciones de investigación que realiza la Universidad de San Carlos de Guatemala; además, formalizar la colaboración con la iniciativa privada como fincas cafetaleras, que es relevante para la conservación de la biodiversidad de los alrededores de la ciudad (Taugourdeau et al., 2014).

La educación y sensibilización fue evaluada, determinando la inclusión de temas de biodiversidad en el currículo de las escuelas en la ciudad (indicador 22) donde se está implementando la inclusión de estos temas por medio del gobierno central, el gobierno local no tiene participación determinante en los planes de estudio de las escuelas. Sin embargo, de acuerdo al Secretariat of the Convention on Biological Diversity (2012), en otros países (Escocia y Singapur) se han implementado programas informales o programas extra-aula donde se trabajan temas de ecología, ambientales y de biodiversidad.

En el mismo tema, el indicador 23 relacionado a los eventos sobre diversidad biológica es necesario mejorarlo, desarrollando más actividades de educación, capacitación y divulgación sobre la diversidad biológica local. Para hacer efectivo un programa que incluya eventos sobre diversidad biológica es necesario realizar un proceso participativo. El proceso deberá incorporar a los diferentes sectores de la ciudad de La Antigua Guatemala, y de esa manera formular e implementar una Estrategia Local de Diversidad Biológica y su Plan de Acción congruente con las iniciativas del CDB y la Estrategia Nacional de Diversidad Biológica (Avlonitis et al., 2013; Conap, 2012).

En general en este estudio encontramos varias “dificultades" para evaluar el Índice de Diversidad Biológica Urbana, como la falta de datos exactos, aspectos de escala (local-regional) y límites. Todos estos problemas han sido reportados por el trabajo más reciente sobre el índice realizado por Kohsaka y colaboradores
(2013) y en el cual se indican otras sugerencias para mejorar el índice como:

- incorporar la dimensión espacial para el caso de servicios ecosistémicos, además de aumentar el número de servicios a ser evaluados, y

- utilizar un enfoque de gradiente para analizar la diversidad biológica (hecho ya realizado por este estudio) y además utilizar grupos funcionales.

De acuerdo a los resultados de Castillo y colaboradores (2013) la ciudadanía tiene interés en participar en aspectos relacionados a diversidad biológica $\mathrm{y}$ tienen conocimiento sobre el papel que desempeñan en la calidad de vida para las sociedades. El índice no incluye hasta el momento este aspecto y se considera que esto es importante, porque permite sugerir que el índice incorpore en su evaluación del componente III de gobernanza, aspectos de la participación activa de la ciudadanía, pues esta es parte esencial en las relaciones que se establecen entre la sociedad y su entorno.

\section{Agradecimientos}

Al Herbario USCG. A la Dra. Lena Chan, Directora del Consejo de Parques Nacionales de Singapur (responsable del grupo de especialistas del CDB de las Naciones Unidas para implementación del CBI), por proporcionar los manuales actualizados y por el asesoramiento de ella y de su equipo en Singapur. Al CDB por avalar los resultados de esta investigación. A la Dirección General de Investigación, Usac por el financiamiento del Proyecto Digi 4.8.63.2.34., a la Municipalidad de La Antigua Guatemala, en especial al Ing. Vitelio Contreras. A los colaboradores de la investigación: Hugo Enríquez, Astrid Jump, Christian Kraker, Mauricio García, Vivian González y Juan Carlos Nájera.

\section{Referencias}

Avlonitis, G., Doll, C., Galt, R., Mader, A., Moreno-Penaranda, R., Patrickson, S., ... Shih, W. (2013). Local biodiversity strategy and action plan guidelines: An aid to municipal planning and biodiversity conservation. Recuperado de http://collections.unu.edu/eserv/UNU:1494/LBSAP_Guidelines.pdf 
Berghöfer, A. (Coord.). (2011). TEEB Manual for cities: Ecosystem services in urban management. The Economics of Ecosystems and Biodiversity. Recuperado de http://doc.teebweb.org/wpcontent/uploads/Study\%20and\%20Reports/ Additional\%20 Reports/Manual\%20for\%20 Cities/TEEB\%20Manual\%20for\%20Cities_ English.pdf

Boreaux, V., Kushalappa, C. G., Vaast, P., \& Ghazoul, J. (2013). Interactive effects among ecosystem services and management practices on crop production: Pollination in coffee agroforestry systems. Proceedings of the National Academy of Science, 110(21), 8387-8392. doi: 10.1073/ pnas. 1210590110

Castillo, F., García, J., López, A., \& Celada, M. (2013). Los servicios ecosistémicos urbanos en las ciudades de Quetzaltenango y la Antigua Guatemala (Inf-2012-36). Guatemala. Universidad de San Carlos de Guatemala, Dirección General de Investigación, Programa Universitario de Investigación en Recursos Naturales y Ambiente.

Chan, L., Hillel, O., Elmqvist, T., Werner, P., Holman, N., Mader, A., \& Calcaterra, E. (2014). User's Manual on the Singapore Index on Cities' Biodiversity (also known as the City Biodiversity Index). Singapore: National Parks Board, Singapore. Recuperado de https://www.nparks. gov.sg/ /media/nparks-real-content/biodiversity/ singapore-index/users-manual-on-the-singaporeindex-on-cities-biodiversity.ashx?la=en

Comité Asesor Nacional sobre Especies Invasoras. (2010). Estrategia nacional sobre especies invasoras en México, prevención, control y erradicación. México, D.F.: Comisión Nacional para el Conocimiento y Uso de la Biodiversidad, Comisión Nacional de Áreas Protegidas, Secretaría de Medio Ambiente y Recursos Naturales.

Consejo Nacional de Áreas Protegidas. (2011). Fortalecimiento de las Capacidades Institucionales para Abordar las Amenazas Provocadas por la Introducción de Especies Exóticas en Guatemala. Guatemala. Documento técnico No. (79-2010). Recuperado de https://www.cbd.int/invasive/doc/meetings/ isaem-2015-01/DECISION\%20SUPPORT\%20 TOOLS/iasem-guatemala-dst-04-esp.pdf
Consejo Nacional de Áreas Protegidas. (2013). Politica Nacional de Diversidad Biológica (Acuerdo Gubernativo 220 -2011). Estrategia Nacional de Diversidad Biológica y su Plan de Acción (Resolución 01-16-2012). La Década de la Vida y el Desarrollo (Políticas, Programas y Proyectos No. 03 [01-2013]). Recuperado de http://www. chmguatemala.gob.gt/Members/esolorzano/ mis-documentos-2012/estrategia-nacional-de-diversidad-biologica-y-plan-de-accion-2012-2022/ estrategia-nacional-de-diversidad-biologica-y-plan-de-accion-version-hconap.pdf/view

Eggermont, H., Balian, E., Azevedo, J. M., Beumer, V., Brodin, T., Claudet, J., ... Le Roux, X. (2015). Nature-based Solutions: New Influence for Environmental Management and Research in Europe. GAIA, 24(4), 243-248.

Haase, D., Schwarz, N., Strohbach, M., Kroll, F., \& Seppelt, R. (2012). Synergies, trade-offs, and losses of ecosystem services in urban regions: an integrated multiscale framework applied to the Lepzig-Halle Region, Germany. Ecology and Society, 17(3), 1-22. doi: 10.5751/ES-04853-170322

Kohsaka, R., Pereira, H., Elmqvist, T., Chan, L., Moreno-Peñaranda, R., Morimoto, Y., ... Pearsell, G. (2013). Indicators for management of urban biodiversity and ecosystem services: City biodiversity index. In T. Elmqvist, M. Fragkias, J. Goodness, B. Güneralp, P. Marcotullio, R. McDonald, ... C. Wilkinson (Eds.). Urbanization, Biodiversity and Ecosystem Services: Challenges and Opportunities (Chapter 33, pp. 669-718). Netherlands: Springer.

Kraker-Castañeda, C., \& Pérez-Consuegra, S. G. (2011). Contribución de los cafetales bajo sombra en la conservación de los murciélagos de la Antigua Guatemala, Guatemala. Acta Zoológica Mexicana, 27(2), 291-303.

López García, J.S. \& Martín Hernández, M.J. (2010). Plan Maestro de La Antigua Guatemala, República de Guatemala. Universidad de Las Palmas de Gran Canaria, Consejo Nacional para la Protección de La Antigua Guatemala y Municipalidad de La Antigua Guatemala. 
Martín-López, B., Iniesta-Arandia, I., García-Llorente, M., Palomo, I., Casado-Arzuaga, I., García, D., ... Montes, C. (2012). Uncovering ecosystem service bundles through Social preferences. PLoS ONE, 7(6), 1-11. doi: 10.1371/journal. pone. 0038970

Martins, V., Haddad, C. R., \& Semir, J. (2011). Responses of the invasive Ricinus communis seedlings to competition and light. New Zealand Journal of Botany, 49(2), 263-279. doi: 10.1080/0028825X.2010.548069.

Quiroz, C., Pauchard, N., Marticorena, Al \& Cavieres, L. (2009). Manual de plantas invasoras del centro-sur de Chile (Proyecto CONICIYT PFB-23). Concepción, Chile: Universidad de Concepción, Laboratorio de Invasiones Biológicas,. Recuperado de http://www.academia.edu/13481327/ Manual_de_Plantas_invasoras_del_centro-sur_ de_Chile

Raudsepp-Hearne, C., Peterson, G. D., \& Bennett, E. M. (2010). Ecosystem service bundles for analyzing tradeoffs in diverse landscapes. Proceedings of the National Academy of Science 107(11), 52425247. doi: 10.1073/pnas.0907284107
Secretariat of the Convention on Biological Diversity. (2012). Cities and Biodiversity Outlook. Montreal, Canada: Autor. Recuperado de https://www.cbd. int/doc/publications/cbo-booklet-2012-en.pdf

Taugourdeau, S., Le Maire, G., Avelino, J., Jones, J. R., Ramírez, L. G., Jara, M., ... Roupsard, O. (2014). Leaf area index as an indicator of ecosystem services and management practices: An application for coffee agroforestry. Agriculture Ecosystems \& Environment, 192, 19-37. doi: 10.1016/j.agee.2014. 03.042.

Trzyna, T. (2014). Urban protected areas: Profiles and best practice guidelines (Best Practice Protected Area Guidelines Series No. 22). Gland, Switzerland: IUCN. Recuperado de https://cmsdata.iucn. org/downloads/bpg_urban_protected_areas.pdf

United Nations. (2012). World urbanization prospects: The 2011 Revision. Recuperado de http://www. un.org/en/development/desa/population/publications/pdf/urbanization/WUP2011_Report.pdf 
Anexo 1.

Cálculos para la generación y calificación de los indicadores del índice de diversidad biológica urbana de la ciudad de La Antigua Guatemala

Componente I. Diversidad biológica nativa la ciudad

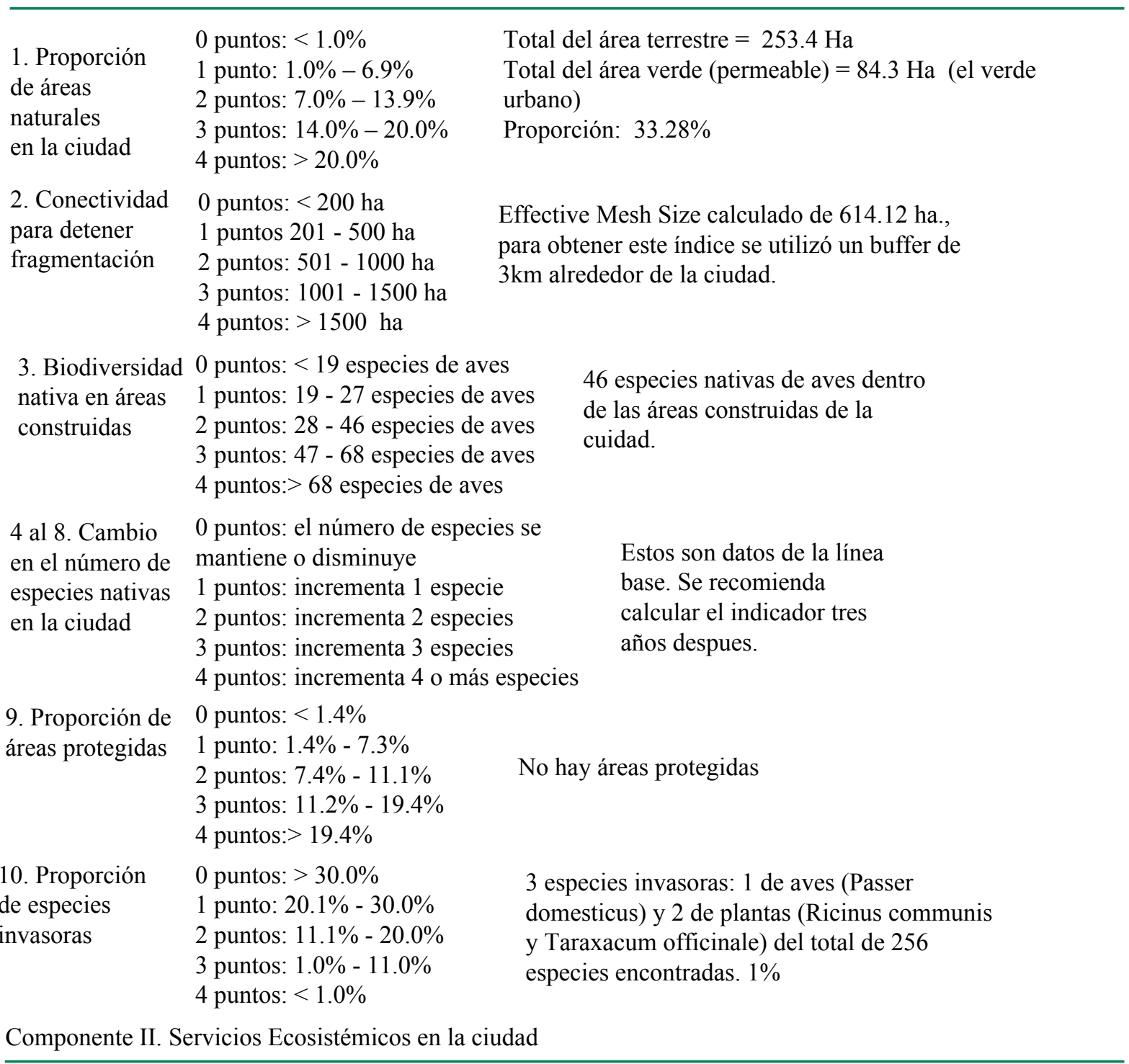

11. Regulación
del agua

0 puntos: $<33.1 \%$

1 punto: $33.1 \%-39.7 \%$

2 puntos: $39.8 \%-64.2 \%$

3 puntos: $64.3 \%-75.0 \%$

4 puntos: $>75.0 \%$
Total del área terrestre $=253.4 \mathrm{Ha}$

Total del área verde (permeable) $=84.3 \mathrm{Ha}$

Proporción: $33.28 \%$ 


$$
\begin{array}{ll}
\text { 12. Regulación } & 0 \text { puntos: }<10.5 \% \\
\text { climática y } & 1 \text { punto: } 10.5 \%-19.1 \% \\
\text { secuestro de carbono } & 2 \text { puntos: } 19.2 \%-29.0 \% \\
& 3 \text { puntos: } 29.1 \%-59.7 \% \\
& 4 \text { puntos: }>59.7 \%
\end{array}
$$

13. Servicios Área de Parques Naturales por 1000 personas educativos y 0 puntos: $<0.1$ ha $/ 1000$ personas

recreativos 1 punto: $0.1-0.3 \mathrm{ha} / 1000$ personas 2 puntos: $0.4-0.6 \mathrm{ha} / 1000$ personas 3 puntos: $0.7-0.9 \mathrm{ha} / 1000$ personas 4 puntos: $>0.9$ ha/ 1000 personas

14. Servicios Visitas Educativas a Parques Naturales educativos y 0 puntos: 0 visitas educacionales formales/año recreativos 1 punto: 1 visita educacional formal/año 2 puntos: 2 visitas educacionales formales/año 3 puntos: 3 visitas educacionales formales/año 4 puntos: $>3$ visitas educacionales formales/año

$$
[273,452 \text { (celdas) } * 0.52] \div(253,400) \times 100=
$$$$
(68,363 \mathrm{~m} 2 / 253,400 \mathrm{~m} 2) \times 100=26.97 \%
$$

Existen 2.37 Has ó 23,718.065297 m2 de parques naturales (pero sin área natural sino con vegetación manejada) 52,700 Habitantes. 0.045 has por cada mil habitantes.

No se realizan visitas a áreas protegidas en el año

Componente III. Gobernanza y manejo de la diversidad biológica en la ciudad

$\begin{array}{lll}\text { 15. Presupuesto } & 0 \text { puntos: }<0.4 \% & \text { Monto destinado a biodiversidad) } \div \\ \text { destinado a } & 1 \text { punto: } 0.4 \%-2.2 \% & \text { (Presupuesto total de la ciudad) } \times 100 \% \\ \text { diversidad } & 2 \text { puntos: } 2.3 \%-2.7 \% & \text { Mayor a 3.7\% (para temas ambientales en } \\ \text { biológica } & 3 \text { puntos: } 2.8 \%-3.7 \% & \text { general) } \\ & 4 \text { puntos: }>3.7 \% & \end{array}$

16. Número de proyectos sobre biodiversidad implementados por las autoridades de la ciudad

17. políticas, reglas $y$ regulaciones-estrategias y planes de acción locales sobre biodiversidad
0 puntos: $<12$ programas/proyectos 1 punto: 12 - 21 programas/proyectos 2 puntos: 22 - 39 programas/proyectos 3 puntos: 40 - 71 programas/proyectos 4 puntos: $>71$ programas/proyectos

0 puntos: No LBSAP

1 punto: LBSAP no se alinea a NBSAP 2 puntos: LBSAP incorpora elementos de NBSAP, pero no incluye ninguna iniciativa del CBD 3 puntos: LBSAP incorpora elementos de NBSAP, e incluye 1 a 3 iniciativas del CBD 4 puntos: LBSAP incorpora elementos de NBSAP, e incluye 4 o más iniciativas CBD
Menos de 12 por año

No se tiene implementado nada.
18. Capacidad institucional
0 puntos: Sin funciones

1 punto: 1 función

2 puntos: 2 funciones

3 puntos: 3 funciones

4 puntos: $>3$ funciones
Vivero municipal 
19. Capacidad institucional
0 puntos: 1 o 2 agencias coopera en asuntos sobre biodiversidad 1 punto: 3 agencias coopera en asuntos sobre biodiversidad 2 puntos: 4 agencias coopera en asuntos sobre biodiversidad 3 puntos: 5 agencias coopera en asuntos sobre biodiversidad 4 puntos: Más de 5agencias coopera en asuntos sobre biodiversidad

20. Participación y asociación

21. Participación y asociación

22. Educación y sensibilización

23. Educación y sensibilización
0 puntos: No tiene procesos de rutina formales o informales de consulta y participación 1 punto: Procesos formales o informales están siendo considerados como parte del proceso rutinario.

2 puntos: Procesos formales o informales están siendo considerados como proceso de rutina 3 puntos: Procesos formales e informales están en proceso de ser implementados como parte del proceso rutinario 4 puntos: Procesos formales o informales existen como parte del proceso rutinario.

0 puntos: No hay consorcios formales/ informales 1 punto: La ciudad en consorcio con 1 - 6 otras agencias nacionales o subnacionales /empresas privadas/ONG/ instituciones académicas/organizaciones internacionales 2 puntos: La ciudad en consorcio con 7-12 otras agencias 3 puntos: La ciudad en consorcio con 13-19 otras agencias 4 puntos: La ciudad tiene convenios con 20 otras agencias

0 puntos: Biodiversidad o sus elementos no están incluidos en el curriculum de las escuelas

1 punto: Biodiversidad o sus elementos son considerados para incluirlos en el curriculum de las escuelas

2 puntos: Biodiversidad o sus elementos se ha planificado incluirlos en el curriculum de las escuelas 3 puntos: Biodiversidad o sus elementos están en proceso de implementación en el curriculum de las escuelas 4 puntos: Biodiversidad o sus elementos están incluidos en curriculum delas escuelas

0 puntos: 0 eventos de divulgación /1000 personas/año 1 punto: 1 - 59 eventos de divulgación /1000 personas/año 2 puntos: 60 -149 eventos de divulgación $/ 1000$ personas/año 3 puntos: 150-300 eventos de divulgación /1000 personas/año 4 puntos: $>300$ eventos de divulgación /1000 personas/año
Más de 5.

Existen

procesos

formales

Entre 7 a 12

instituciones.

Aparentemente
varias
instituciones
están tratando de
impulsar el tema
para el
curriculum
nacional de base.

De 60 -149 eventos

realizados por $/ 1000$

personas/año 